\title{
Telecardiology Assistance in Senior Living Houses
}

\author{
Milica Kaladjurdjevic \\ School of Pharmacy, University of Camerino, Telemedicine and Telefaramacy Department, Camerino62032, Italy
}

\begin{abstract}
The CHF (chronic heart failure) represents one of major cause of mortality and morbidity in developed countries. It is expected that incidence of heart failure associated with aging, will rise exponentially, due to its higher prevalence among population over 75 years. The SLH (senior living house) is missing an adequate secondary healthcare assistance in order to provide a qualitative secondary prevention to the resident. We have conducted a pilot project of telecardiology in one senior living house in 2013, with duration of 6 months; we decided to expend the project as an observational trial with primary objective to evaluate impact of telemedicine on secondary prevention of clinical cardiovascular outcomes/hospitalization in senior living house setting and secondary objectives to evaluate quality of life using Minnesota living with Heart Failure questionnaire and to evaluate cost savings and additional benefits earned by telemedicine. For the study, $85 \%$ powered at the $5 \%$ level of significance; to detect differences, we need 310 patients, including $10 \%$ drop out. As results, during the pilot project, 23 patients with median age of 86 years were monitored; we are currently enrolling new senior living house and patients with CHF that resides in senior living house.
\end{abstract}

Key words: CHF, telemedicine, Telecardiology, secondary prevention, elderly.

\section{Introducton}

The CHF (chronic heart failure) is one of principle causes of re-hospitalization, in population over 75 years of age. With future aging process, the prevalence of cardiovascular disease will rise exponentially. The ratio of healthcare spending for elderly population is already 4 fold higher versus healthcare spending for population under 65 years old. In near future, the elderly will represent one fourth (1/4) of total population. Considering the aging process and associated increase of chronic disease prevalence in next future, healthcare systems will face financial and professional constraints in provision of qualitative and equally accessible healthcare services.

The chronic heart failure represents one of major causes of mortality and morbidity in industrialized countries. The incidence of heart failure is estimated from 1 to 5 cases per 1,000 population with exponential increase in the population over 65 old, because of what heart failure is considered a disease of aging. High incidence of re-hospitalization due to

\footnotetext{
Corresponding author: Milica Kaladjurdjevic, Ph.D., research fields: telemedicine. E-mail: milica.kaladjurdjevic@unicam.it.
}

frequent CHF exacerbation makes this group of patients among the highest consumer of healthcare and social services.

Secondary prevention is an important gatekeeper of hospitalization and over consumption of tertiary care service. Secondary prevention can detect early signs of organ worsening and prevent further deterioration, by intervening with adequate treatment. In order to detect early symptoms, it is necessary to monitor frequently patient's vital signs.

Telemedicine showed positive results in regards of secondary prevention. We posed the question whether the telecardiology assistance in long term trial of 24 months, on large sample can show decrease in re-hospitaliaztion and improvement of quality of life. The article aims at presenting a work in progress project, the methodology used in development of the project, the problems that have been encountered and further perspectives. The quality of data transmitted still represent a limitation for telemedicine wider use. We present the Chronic Heart Failure as a future public healthcare problem by introducing telecardiology assistance in SLH (senior living house) and our project pilot. Finally, we present a protocol 
developed on the basis of project pilot results. Major results are not available yet.

\section{Chronic Heart Failure-Future Public Healthcare Impact}

The heart failure represents a terminal syndrome of heart function deterioration, often affected by number of pathology associated with process of aging. The heart insufficiency has an important effect on long life perspective and quality of life characteristics as capacity to perform everyday activity.

Quality of life and social activity are in relation with patient's mobility. With aging, the level of disability is increasing; according to Global Burden of Disease Study, cardiovascular diseases will generate $18\left(10^{3}\right)$ DALY (disability adjusted life years) by 2020 [1]. The prevalence of CHF increases with age: $2 \%$ for persons from 40 to 59 years, more than $5 \%$ for patients between 60 and 69 years, and $10 \%$ for persons more than 70 years old [2]. A heart failure is considered a cause of $20 \%$ of all re-hospitalization generated by patient's over 65 year old.

The cost of heart failure care is estimated as percent of total healthcare spending equal to $13 \%$, with the highest cost spent for hospital care, which represents $77 \%$ of total healthcare spending for heart failure [3]. The major causes of re-hospitalization are non adherence to treatment and non adherence to physician advices [4].

These issues are minor causes of re-hospitalization when patients reside in senior living house, where they are assisted by a nurse. Still senior living house requires cardiology secondary care consultation and additional monitoring of cardiovascular patients. These additional support provided by telemedicine at distance would prevent worsening of patient health status and frequent re-hospitalization, by early symptoms detection and optimization of therapy.

\section{Senior Living House - Italy}

A SLH are residential structure for elderly who is partially or completely non auto-sufficient. The primary function of a SLH is to guarantee physical health and psychological well being. A SLH provides a medical geriatric, nurse and rehabilitation assistance; however, there is a lack of secondary care prevention assistance. Therefore, we want to address an added value of telemedicine in SLH setting, characterized with elderly population mostly affected by cardiovascular diseases, who are in a need for monitoring and secondary cardiology prevention assistance. The percentage of elderly population who resides in SLHs varies by country. The secondary care and tertiary care services provided to the residents of a SLH are reimbursed by national healthcare insurance.

The presence of healthcare professionals, as nurses, in SLHs was important for our project as it facilitated communication between Cardiology Department and SLH. A SLH, usually, has a medical doctor, a nurse, and caregivers; the specialized assistance as cardiologic consultation may be an additional benefit in order to improve the quality of care provided.

\section{Social Inclusion-Role of Telemedicine and Previous Clinical Trials of Telemedicine in $\mathrm{CHF}$ patient}

A distance clinical monitoring system combining telecommunication technology and medical device for transmission of vital signs measurement can decrease readmission rate and improve social well being $[5,6]$.

Telemedicine can offer monitoring of ECG and blood pressure at distance, early detection of symptoms and signs, and secondary prevention through optimization of therapy. Optimization of therapy is represented by real-time titration and adequate dosing of medication through tight follow-up of the patient symptoms. In previous years, research has shown that tight home care monitoring improves functional status of the elderly [7]; the trial conducted by Kornowski [8], who evaluated a weekly home visit by internist specialist, has shown a significant decrease of readmission to the hospital, improved 
functional status of the patient, with consequent decrease of healthcare expenditure.

\section{Clinical Trail Proposal}

The project pilot has been conducted in 2013, with duration of 6 months. During 6 months period, 23 patients affected by cardiovascular disease have been enrolled, after the verbal inform consent has been given by their family member. The project pilot has shown limitations in regard of telemonitoring frequency and readability of ECG.

ECG readability is very important for accurate recognition of ECG changes from its baseline. We have faced a problem of interferences around the patient (cell phone, TV, vibration from bedsore mattress) which intervened with morphology of ECG line. The readability of ECG has been improved after pilot project; we added additional filters that improved readability and accuracy of ECG.

During the pilot project, the average age of patient enrolled was 86; considering Italian longevity, this population is considered very old. During the pilot project, we had seven hospitalizations for non cardiac diagnosis, with length of stay of 76 days cumulative. The number of re-hospitalization for CHF was 2, with the stay length of 2 days and with mortality rate during re-hospitalization of $100 \%$.

The pilot project had the objective to evaluate feasibility of telecardiology, to understand the problems of technology and to evaluate professionals' and patients' responsiveness to the telecardiology. The patients enrolled in pilot project were affected by any type of cardiopathy, those affected by $\mathrm{CHF}$ represented $50 \%$ of patients included in pilot project.

The adherence to the guidelines for CHF treatment is one of prerogative of adequate treatment. We evaluated if every patient affected by CHF (10 patients) was using beta blockers and diuretics. In further extended trial, we will observe change in therapy and the need for optimization of therapy, taking in consideration co-morbidities associated with CHF.

\subsection{Extended Clinical Trail}

As prevalence of CHF increases with age, the population over 75 years of age was considered as target group population, where secondary prevention via telecardiology would demonstrate a positive effect Primary Objectives: To evaluate impact of telemedicine on secondary prevention and reduction of clinical cardiovascular outcomes/hospitalization in senior living house setting;

Secondary Objectives: To evaluate quality of life using MlwHF (minnesota living with heart failure) and cost-effectiveness.

\subsection{Definition of Patients}

The patients were over 75 years old who live in a SLH and with NYHA (New York Heart Association) II and III, but not on dialysis.

We choose over 75 years of age, as the prevalence of CHF and re-hospitalization rate is higher in this population, hence this group will benefit more from secondary prevention than younger group of population.

The patients living in a SLH affected with CHF have nurse assistance; additionally, with trial, we will provide a frequent and continuous monitoring and pharmaceutical - drug titration adjustment provided by cardiologist via telemedicine.

Inclusion Criteria: Patients with heart failure diagnosis based on guidelines ESC (European Society of Cardiology); Patient over 75; Patient NYHA II - III; Patient with ischemic and valvular heart pathology; hypertensive and dilatative cardiomyopathy; $\mathrm{E} F=$ $35 \%$

Exclusion Criteria: Patients below 75 years; Patient affected by psychiatric disease and dementia; Patient on dialysis; Cahexia; Non cooperative patient.

\subsection{Study Design}

An observational trial, have a duration of 24 months. The telemedicine monitoring will have frequency of weekly vital parameters measurement (blood pressure, oxygen saturation, weight and ECG). In a previous 
pilot project, we did not observe weight measurement changes, as most of patients were very old (86 years, average) and were bedridden. Variation in body weight is an important diagnostic parameter of $\mathrm{CHF}$ symptomatology.

The design of trial does not include the control group. The rate of re-hospitalization in population over 75 years old, affected by CHF is established from previous large clinical trails. Results from this trial will be compared with results established by large registries for CHF (OPTIMIZE, ADHERE) [9-11].

\subsection{Sample Size Calculation}

The previous studies conducted using randomized clinical trial design showed a decrease in readmission of $20-40 \%$ with telemedicine [12-17].

The difference expected is at least $15 \%$ decrease of hospital readmission. For the study, $85 \%$ powered at the $5 \%$ level of significance, to detect difference we need 310 patients including 10\% drop out. Cumulative probability and survival curves will be constructed using Kaplan-Meier estimates [18] and compared using the Log-Rank test [19]. The rate of hospital readmission, i.e., HR (hazard ratio) [19] and 95\% CI (confidence interval) will be calculated using a Cox regression model [20]. Probability values $<0.05$ will be considered significant.

\subsection{Subgroups}

Elderly population is affected by multi-pathology, the CHF causes reduced perfusion of other organs. Inadequate perfusion has a negative impact on an organ's function, altering its function. We want to evaluate the effect of secondary prevention for CHF, and its secondary effect on organ functional preservation. Therefore, the next subgroup evaluation will be conducted: 1 . Diabetes vs. no Diabetes; 2 . Hypertension vs. no Hypertension; 3. Ischemic CM vs. no Ischemic CM; 4. B blockers vs. no B blockers; 5. Dose of loop diuretic (40 mg vs. $>40 \mathrm{mg} /$ day).

The co-morbidities represented in subgroups are most frequent co-morbidities, related to structural and functional organ's modification, associated with aging process. We did not establish a number of patients that would be necessary for each group, to demonstrate significant results among the groups. If during the trial a difference between groups in regard of primary objective (re hospitalization) will be observed, further adjustment of design will be done in order to demonstrate significant results in subgroups.

The long term therapy with diuretics usually causes drug resistance, which requires an increase of drug dosage in order to obtain the same effect. We want to evaluate the effect of combined tight monitoring and secondary prevention via telemedicine on lower dosage of diuretics vs. higher dosage of diuretics consumption.

\subsection{Telemedicine Equipment}

A consolidated platform for telemedicine that

Table 1 Summary on the pariticipants.

\begin{tabular}{|l|l|l|l|l|}
\hline Pilot project in short figures & Average age & Intervention & $\begin{array}{l}\text { Number } \\
\text { teleconsultation }\end{array}$ \\
\hline No. of patients & Patients with CHF & $\begin{array}{l}\text { ofG } \\
\text { PA } \\
\text { Saturation }\end{array}$ & 510 \\
\hline 23 & 10 & 86.7 & \\
\hline
\end{tabular}

Table 2 Details on CHF therapy.

\begin{tabular}{|c|c|c|c|c|}
\hline \multicolumn{5}{|c|}{ Usage of CHF therapy, represented in pilot project } \\
\hline Beta blocker & Diuretics & $\begin{array}{l}\text { Antitrombotics, } \\
\text { Anticoagulant } \\
\text { Clexane, Cardioaspirina, } \\
\text { Plavix, Arixtra, warfarin } \\
\end{array}$ & Statins & Inotropic drugs \\
\hline $100 \%$ & $100 \%$ & $100 \%$ & $8 \%$ & $8 \%$ \\
\hline
\end{tabular}


supports multi-specialty tele-consultations is used. The technical system needs only to be configured at the moment of deployment to the user and is really easy to use. The software allows real-time and storage and forward data sending to manage different need of use and possible lacks of connectivity without data loss. It integrates different types of medical devices tailored for the nurse use. The cardiologist communication and consultation are supported with storing, reporting, real-time communication and high resolution visualization of the documents. Every kit contains medical device (blood pressure device, oxygen saturation device, weight control device, ECG -12 leads, electronic stethoscope), and a tablet with easy to use software.

\subsection{Intervention}

A nurse once a week, usually in the morning, would perform measurements of vital signs, and send them to cardiology department; after clicking on the button send, nurse receives the message that measurement has been sent successfully. The telemedicine kit is very easy to use; after the access to the Internet, the software application opens instantly, and displays 4 images, representing the access to separate pages of 4 vital signs measurement (saturation, blood pressure, weight, and ECG); all data measured from medical devices are acquired by the software installed on a tablet, via a Bluetooth connection. When data are transmitted from medical devices to software, they will appear in defined boxes on display, the nurses have to control if data transmitted on tablet correspond to data obtained by the medical device, afterwards the nurses have to push button send and wait until a message that confirms that data have been sent successfully appears. This procedure is repeated for each of four measurements.

Every morning, one of cardiologists at cardiology ward accesses the software application by logging with his username and password, the application will open by displaying a list of patients enrolled in the trial.

When clicking on patient name and choosing clinical data option, the patient's page will appear, it contains all measurement sent by the user, with date of the performed measurement. The cardiologist has the opportunity to consult previous measurements and to visualize all data graphically; graph contains all changes in patient's vital signs from the first measurement.

\subsection{Quality of Life Evaluation and Cost Effectiveness Analysis}

The Minnesota living with heart failure questionnaire will be used for evaluation of quality of life improvement. The Minnesota living with heart failure questionnaire is a patient self-assessment measurement, being developed to evaluate the therapeutic response to intervention for heart failure [13].

\section{Results}

During the project pilot, 23 patients affected by cardiovascular disease who were living in a SLH were monitored three times per week for the period of 6 months. The results showed limitation in regard to the readability of ECG and medical doctors' culture about telemedicine. After conclusion of pilot project on the end of 2013, these issues were addressed. Then, the clinical trial protocol presented in this paper was developed.

On March 2014, we started with selection of new SLH and consultation with senior living house board managers, in order to establish their interest and their point of view regarding benefits of telemedicine service in SLH setting. The consultation process has been concluded by the recipient with positive feedback in regard to our request to conduct our project at SLH. In June 2014, we have presented telemedicine platform and telemedicine kit, with following telemedicine training to health professionals at SLHs [7]. 
From June 2014 to September 2014, SLH nurses and medical doctors had opportunity to test the platform and kits in order to evaluate, amount of time necessary for measurements and its process of sending to cardiology department. On September 2014, we received written response of their consent to participate at the project and to use telemedicine platform. We are at the moment enrolling the patients with CHF who are living in a SLH, as well we are enrolling new SLH located in Region Marche, Italy. Major results are not still available.

\section{Conclusion}

The promise of technology development allows a healthcare system to provide service in a simplified manner from a distance.

The telemedicine allows continuity of care, versus episodic encounters between patients and physician seen in usual care, and better secondary prevention. A lack of large clinical trials on telemedicine and healthcare system's rigid attitude towards interventions with scarce evidence based results, are the reason why telemedicine is not widely used for monitoring and consulting of elderly population.

We believe that secondary prevention intervention provided through cardiology department to institution where elderly population resides may permit better healthcare services for elderly population wherever they live.

\section{References}

[1] Murrey, C. J. L., and Lopez, A. D. 1997. "Alternative Projection of Mortality and Disability by Cause 1990-2020: Global Burden Disease Study." Lancet 349: 1498-504.

[2] Rozzini, R., Sabatini, T., Costa, T., and Trabucchi, M. 2004. "Il Trattamento Dello Scompenso Cardiaco Nell'anziano: Modelli Di Cura a Confronto." Geriatria \& Pratica Medica 2: 8-10.

[3] Politi, C., Deales, A., Cicchitelli, F., Marcobelli, A., Barbadoro, P., Zorzan, R., and Stanislao, F. D. 2006. "Analisi dei costi sanitari per lo scompenso cardiaco nella regione Marche." PharmacoEconomics-Italian Research Articles 3: 165-75.
[4] Lenarda, A. D. 2003. "Current Presentation and Management of Heart Failure in Cardiology and Internal Medicine Hospital Units: A Tale of Two Worlds-The TEMISTOCLE Study." American Heart Journal (October).

[5] Shah, N. B., Der, E., Ruggerio, C., Heidenreich, P. A., and Massie, B. M. 1998. "Prevention of Hospitalizations for Heart Failure with an Interactive Home Monitoring Program.” Am Heart J. 135: 373-8.

[6] Rich, M. W. 1999. "Heart Failure Disease Management: A Critical Review." Journal of Cardiac Failure 5: 64-75.

[7] Antonicelli, R. 2008. "Impact of Telemonitoring at Home on the Management of Elderly Patients with Congestive Heart Failure." J .Telemed Telecare 4: 300-5.

[8] Kornowski, R. 1995. "Intensive Home-Care Surveillance Prevents Hospitalization and Improves Morbidity among Elderly Patients with Severe Congestive Heart Failure." Am Heart J. 129: 762-6.

[9] Rossi, S. J. 2008. "Influence of Coronary Artery Disease and Coronary Revascularization Status on Outcomes in Patients with Acute Heart Failure Syndromes: A Report from OPTIMIZE-HF (Organized Program to Initiate Lifesaving Treatment in Hospitalized Patients with Heart Failure)." European Journal of Heart Failure 10: 1215-23.

[10] Mentz, R. J. 2012. "Clinical Characteristics and Outcomes of Hospitalized Heart Failure Patients with Systolic Dysfunction and Chronic Obstructive Pulmonary Disease: Findings from OPTIMIZE-HF." European Journal of Heart Failure 14: 395-403.

[11] Adams, K. F. J. 2005. "Characteristics and Outcomes of Patients Hospitalized for Heart Failure in the United States: Rationale, Design, and Preliminary Observations from the First 100,000 Cases in the Acute Decompensated Heart Failure National Registry (ADHERE)." American Heart Journal 149 (February): 209-16.

[12] Clark, R. A., Inglis, S. C., McAlister, F. A., Cleland, J. G. F., and Stewart, S. 2007. "Telemonitoring or Structured Telephone Support Programmes for Patients with Chronic Heart Failure: Systematic Review and Meta-Analysis.” BMJ 334 (April): 942-5.

[13] Cleland, J. G. F., Louis, A. A., Rigby, A. S., Janssens, U., and Balk, A. H. M. M. 2005. "Noninvasive Home Telemonitoring for Patients with Heart Failure at High Risk of Recurrent Admission and Death the Trans-European Network-Home-Care Management System (TEN-HMS) Study." Journal of the American College of Cardiology 45 (10): 1654-64.

[14] Dendale, P. 2012. "Effect of a Telemonitoring-Facilitated Collaboration between General Practitioner and Heart Failure Clinic on Mortality and Rehospitalization Rates in 
Severe Heart Failure: The TEMA-HF 1 (TElemonitoring in the Management of Heart Failure) Study." European Journal of Heart Failure 14: 333-40.

[15] Rector, T. S., Kubo, S. H, and Cohn, J. N. 1987. "Patients' Self-Assessment of Their Congestive Heart Failure. Part 2: Content, Reliability and Validity of a New Measure, the Minnesota Living with Heart Failure questionnaire." Heart Failure 28: 198-209.

[16] Hall, M. J., Levant, S., and DeFrances, C. J. 2012. "Hospitalization for Congestive Heart Failure: United States, 2000-2010.” NCHS Data Brief 108 (October): 1-8.

[17] Efron, B. 1988. "Logistic Regression, Survival Analysis, and the Kaplan-Meier Curve." Journal of the American Statistical Association 83: 414-25.

[18] Matthews, D. E., and Farewell, V. T. 2007. "Using and Understanding Medical Statistics.” Basel, S. Karger AG, 67-75. doi: 10.1159/000099422.

[19] Fisher, L. D., and Lin, D. Y. 1999. "Time-Dependent Covariates in the Cox Proportional-Hazards Regression Model." Annual Review of Public Health 20 (May): 145-57.

[20] Lin, D. Y., 1994. "Cox-regession Analysis of Multivariate Failure Time Data: The Marginal Approach.” Statistics in medicine 13: 2233-47. 